\title{
Pien Tze Huang suppresses VEGF-C-mediated lymphangiogenesis in colorectal cancer
}

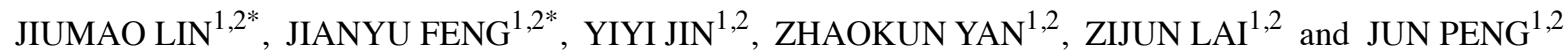 \\ ${ }^{1}$ Academy of Integrative Medicine, and ${ }^{2}$ Fujian Key Laboratory of Integrative Medicine on Geriatrics, \\ Fujian University of Traditional Chinese Medicine, Fuzhou, Fujian 350122, P.R. China
}

Received August 8, 2016; Accepted September 22, 2016

DOI: $10.3892 / o r .2016 .5186$

\begin{abstract}
Colorectal cancer (CRC) is one of the most common malignancies worldwide. The majority of patients are not suitable for surgery due to the presence of metastatic disease at the time of diagnosis, which has led to a high mortality rate for patients with CRC. Lymphangiogenesis, formation of new lymphatic vessels, plays an critical role in cancer progression particularly in cancer metastasis. Vascular endothelial growth factor-C (VEGF-C) has been previously demonstrated to play a pivotal role in cancer metastasis and therefore has become an attractive target for anticancer treatments. Pien Tze Huang $(\mathrm{PZH})$ is a well-known traditional Chinese formula, which has exhibited significant therapeutic effects against CRC. However, the molecular mechanisms underlying its anticancer effects, particularly in regards to antimetastasis activity, still require further elucidation. In the present study, we evaluated the effects of $\mathrm{PZH}$ on cell migration and VEGF-C expression using various human $\mathrm{CRC}$ cell lines. Moreover, using a VEGF-Cstimulated human lymphatic endothelial cell (HLEC) model, we demonstrated that PZH suppresses lymphangiogenesis by attenuating cell migration and tube formation. This indicates that $\mathrm{PZH}$ possesses significant antimetastatic activity. Moreover, suppression of lymphangiogenesis by $\mathrm{PZH}$ via the downregulation of VEGF-C may be a potential molecular mechanism by which PZH inhibits metastasis in CRC.
\end{abstract}

Correspondence to: Professor Jun Peng, Academy of Integrative Medicine, 1 Qiuyang Road, Minhou Shangjie, Fuzhou, Fujian 350122, P.R. China

E-mail: pjunlab@hotmail.com

${ }^{*}$ Contributed equally

Abbreviations: PZH, Pien Tze Huang; CRC, colorectal cancer; HLEC, human lymphatic endothelial cell; LV, lymphtic vessel; VEGF-C, vascular endothelial growth factor-C

Key words: Pien Tze Huang, colorectal cancer, lymphangiogenesis, VEGF-C, metastasis

\section{Introduction}

Colorectal cancer (CRC) is the third most commonly diagnosed cancer and one of the leading causes of cancer-related death worldwide with a rising annual incidence in numerous countries except the USA (1). Conventional therapies including surgery, chemotherapy or radiotherapy have been shown to be relatively ineffective (2). The main reasons for the lack of efficacy of these therapies are regional lymph node and/or distal metastasis of CRC cells (3). Approximately one quarter of CRC patients present with metastatic disease at the time of diagnosis, and their 5-year survival rate is near $10 \%$ (4). Therefore, elucidating the mechanisms involved in the metastatic cascade underlying this malignancy is a key goal for developing therapeutic agents to reduce CRC-associated mortality.

Lymphangiogenesis or the growth of lymphatic vessels(LVs), is an important step in tumor progression that supports tumor growth and metastatic dissemination (3). Lymphangiogenesis and early regional metastasis frequently occurs in several types of malignant tumors including CRC (5). The density of LVs in or surrounding tumors is closely associated with lymph node metastasis and prognosis of CRC patients (3). Therefore, metastasis to regional lymph nodes is generally believed to be the first indication that a tumor has progressed to metastatic competence (6). Thus, tumor lymphangiogenesis may be a crucial target of antimetastatic agents.

In the past decade, there has been a dramatic increase in the number of studies examining mechanisms of tumor-associated lymphangiogenesis and lymphatic metastasis. Vascular endothelial growth factor-C (VEGF-C), a member of the VEGF family, was the first lymphangiogenic growth factor to be identified (5). Mechanistically, the binding of VEGF-C to its cognate receptor VEGFR-3, which is expressed on human lymphatic endothelial cells (HLECs) (6), promotes proliferation and migration of HLECs as well as the formation of LVs $(7,8)$. Thus, upregulation of VEGF-C expression has been implicated in induction of tumor lymphangiogenesis and lymphatic invasion (9). Therefore, targeting VEGF-C could be a novel anti-lymphangiogenic therapy.

Genomic instability and heterogeneity of tumor cell populations can lead to a shift in the expression of angiogenic factors during tumor progression. Various tumors at their advanced stage express multiple angiogenic factors (10). During this late stage, anti-VEGF-C therapy is predicted to be ineffective 
or even encounters drug resistance. Indeed, it is possible that anti-VEGF-C therapy may even allow tumors to switch on other angiogenic pathways for lymphangiogenesis. As a result, tumors can escape from anti-VEGF-C therapy after a relative long-term treatment. Additionally, drugs used in anti-VEGF-C therapy can lead to an increase in side-effects such as rash, diarrhea, hypertension, gastrointestinal perforation and arterial thromobembolic events (11). These problems highlight the urgent need for the development of more effective anticancer agents.

Pien Tze Huang (PZH) is a well-known traditional Chinese formulation that was first prescribed 450 years ago by a royal physician in the Ming Dynasty. The main active ingredients of PZH include: Moschus, Calculus Bovis, Snake Gall and Radix Notoginseng. These ingredients together confer PZH properties of heat-clearing, detoxification, promotion of blood circulation and removal of blood stasis (12). Since in the Chinese medicine system accumulation of toxic dampness and heat is one of the major causative factors in the pathogenesis of cancers, $\mathrm{PZH}$ is thought to be an effective anticancer agent In fact, $\mathrm{PZH}$ has long been used as an alternative treatment strategy for cancers in China and Southeast Asia. Previously, we reported that $\mathrm{PZH}$ can inhibit CRC growth in vivo and in vitro via promotion of apoptosis and suppression of proliferation of cancer stem cells (CSCs) as well as inhibition of angiogenesis and reversal of multidrug resistance through multiple pathways while causing minimal side-effects (13-17). Recently, we found that $\mathrm{PZH}$ also prevents metastasis by inhibiting the progression of epithelial-mesenchymal transition (EMT) $(18,19)$, but the underlying mechanism of the antimetastasis effects of PZH are still unknown. To further elucidate the mechanism underlying the anticancer activity of $\mathrm{PZH}$, in the present study, we evaluated the effects of $\mathrm{PZH}$ on tumor metastasis and lymphangiogenesis using different CRC cell lines and the VEGF-C-stimulated HLEC model.

\section{Materials and methods}

Materials and reagents. Roswell Park Memorial Institute (RPMI)-1640 medium (C11875500BT), Dulbecco's modified Eagle's medium (DMEM) with high glucose (C11995500BT), fetal bovine serum (FBS; \#10099-141), penicillin-streptomycin (SV30010), 0.25\% trypsin-EDTA (\#25200-072), Pierce RIPA buffer (\#89901), Pierce BCA Protein Assay kit (\#23227) and SuperSignal ${ }^{\mathrm{TM}}$ West Pico Chemiluminescent Substrate (\#34080) were all purchased from Thermo Fisher Scientific, Inc. (Waltham, MA, USA). CellTiter $96^{\circledR}$ AQueous Non-Radioactive Cell Proliferation Assay (MTS; G5430) was provided by Promega Corporation (Madison, WI, USA). The exogenous VEGF-C (CYT-527) was purchased from ProSpec-Tany TechnoGene Ltd. (East Brunswick, NJ, USA). Annexin V-FITC apoptosis detection kit (KGA108) was obtained from KeyGen Biotech Co., Ltd. (Jiangsu, China). Transwell chambers $(8.0 \mu \mathrm{m}$; \#3422) were obtained from Corning Life Sciences (Corning, NY, USA). The In Vitro Angiogenesis assay kit (ECM625) and nitrocellulose (NC) membrane $(0.45 \mu \mathrm{m}$; HATF00010) were purchased from Millipore (Billerica, MA, USA). Rabbit polyclonal antibody against VEGF-C (\#2445), VEGFR-3 (\#2485) and $\beta$-actin (\#4967) were obtained from Cell Signaling Technology
(Beverly, MA, USA). Rabbit polyclonal antibody against MMP-2 (ab37150) and MMP-9 (ab38898) were purchased from Abcam (Cambridge, MA, USA). HRP-conjugated goat anti-rabbit secondary antibody (E030120) was purchased from EarthOx Life Science (Millbrae, CA, USA). Culture flask and plates were purchase from NEST Biotechnology Co., Ltd. (Wuxi, Jiangsu, China). All the other chemicals used, unless otherwise stated, were obtained from Sigma Chemicals (St. Louis, MO, USA).

Preparation of $\mathrm{PZH}$. PZH was obtained from and authenticated by Zhangzhou Pien Tze Huang Pharmaceutical Co., Ltd. (Zhangzhou, China) (Chinese FDA approval no. Z35020242, lot no. 1009039). Stock solutions of PZH were prepared just before use by dissolving the $\mathrm{PZH}$ powder in phosphate-buffered saline (PBS) to a concentration of $20 \mathrm{mg} / \mathrm{ml}$. The working concentrations of PZH were made by diluting the stock solution in the culture medium.

Cell culture. Human metastatic CRC cell lines HCT-8, HCT-116 and SW620 were purchased from the Cell Bank of the Chinese Academy of Sciences (Shanghai, China). The HLEC was purchased from the JENNIO Biological Technology Co., Ltd. (Guangzhou, China). HCT-8, HCT-116 cells and HLECs were grown in RPMI-1640; SW620 cells were grown in DMEM. All cell media were supplemented with $10 \%$ (v/v) FBS, $100 \mathrm{U} / \mathrm{ml}$ penicillin and $100 \mu \mathrm{g} / \mathrm{ml}$ streptomycin, and cultured at $37^{\circ} \mathrm{C}$ with $5 \% \mathrm{CO}_{2}$ in a humidified incubator (Forma 3110; Thermo Fisher Scientific, Inc.).

Treatment of PZH and exogenous VEGF-C. CRC cells were seeded into 6-well plates for $12 \mathrm{~h}$, which was followed by $\mathrm{PZH}$ treatment for the indicated period of time. HLECs were first grown in complete RPMI-1640 (10\% FBS) until $~ 60 \%$ confluency, and then continuously cultured in FBS-free medium overnight. The medium was replaced with RPMI-1640 with $2 \%$ FBS and cells were treated with $5 \mathrm{ng} / \mathrm{ml} \mathrm{VEGF-C} \mathrm{and/or}$ various concentrations of $\mathrm{PZH}$ for the indicated period of time.

Observation of morphological changes. Morphology of HLECs was observed using a phase-contrast microscope (FMIL/DFC295; Leica, Wetzlar, German) after PZH and/or exogenous VEGF-C treatment for $24 \mathrm{~h}$. The images of five randomly selected views were captured at a magnification of $\mathrm{x} 200$.

Cell viability evaluation. Cell viability was assessed using MTS assay. CRC cells and HLECs were seeded in 96-well plates. After PZH and/or exogenous VEGF-C treatment for 24 or $48 \mathrm{~h}, 10 \mu \mathrm{l}$ MTS was added to each well after which the samples were incubated for $1 \mathrm{~h}$ at $37^{\circ} \mathrm{C}$. The resulting absorbance was measured at $490 \mathrm{~nm}$ using an ELISA reader (Model ELx800; BioTek, Winooski, VT, USA).

Detection of apoptosis. After incubation with various concentrations of PZH and/or exogenous VEGF-C for $24 \mathrm{~h}$, apoptosis of HLECs was determined by FACSCalibur and Annexin V-FITC apoptosis detection kit, as previously described (14). Staining was performed according to the manufacturer's instructions. In the present study, 

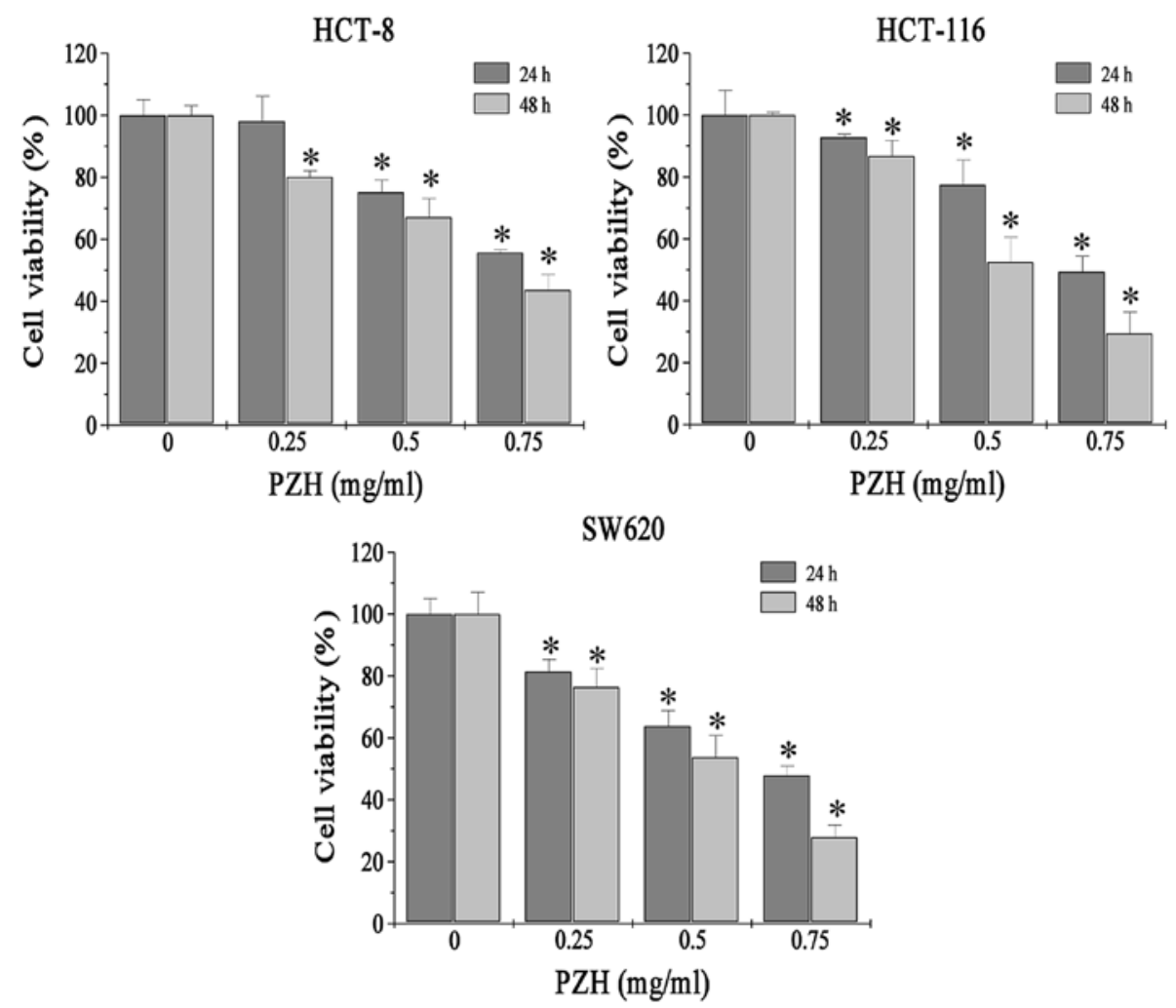

Figure 1. PZH reduces the viability of CRC cell lines. Subsequent to treatment with $0,0.25,0.5$ and $0.75 \mathrm{mg} / \mathrm{ml}$ of PZH for 24 or $48 \mathrm{~h}$, cell viability of human metastatic CRC cell lines HCT-8, HCT-116 and SW620 was determined by MTS assays. The data were normalized to the viability of the control cells. Data are averages with $\mathrm{SD}$ (error bars) from at least three independent experiments; ${ }^{*} \mathrm{P}<0.05$, vs. the untreated control cells.

Annexin V/PI double-negative population (labeled as LL in the FACS diagram) indicates viable cells; Annexin V-positive/PI-negative or Annexin V/PI double-positive population (labeled as LR or UR in the FACS diagram) represents cells undergoing early or late apoptosis, respectively.

Migration assays. Migration ability of the CRC cell lines was evaluated by wound-healing, as previously described (15). Briefly, CRC cells were seeded into 6-well plates $\left(10^{6}\right.$ cells/well in $2 \mathrm{ml}$ culture medium). After $12 \mathrm{~h}$ of incubation, cells were scraped away vertically in each well using a P200 pipette tip. Five randomly selected views along the scraped line were photographed from each well using a phase-contrast inverted microscope at a magnification of $x 100$. Cells were then treated with the indicated concentrations of PZH for $24 \mathrm{~h}$ and another set of images were captured in the same way. A reduction in the scraped area indicates migration of cells. For HLECs, the migration assay was performed using Transwell cell culture chambers as previously described (18). The inserts were placed within a 24-well chamber containing $0.7 \mathrm{ml}$ RPMI-1640 with $10 \%$ FBS to serve as a chemoattractant. As before, HLECs were seeded into 6-well plates and treated with different concentrations of PZH and/or exogenous VEGF-C for $24 \mathrm{~h}$. Cells $\left(5 \times 10^{4}\right.$ cells) were seeded into the inserts suspended in $0.2 \mathrm{ml}$ of serum-free RPMI-1640. The cells were incubated at $37^{\circ} \mathrm{C}$ with $5 \% \mathrm{CO}_{2}$ for $12 \mathrm{~h}$ before the upper surface of the filter was scraped to remove nonmigratory cells. Migrated cells were then fixed and stained with crystal violet. For quantification, the average number of migrating cells/field was assessed by counting five random fields under a phase-contrast microscope at a magnification of $\mathrm{x} 200$.

Tube formation assays. The HLEC tube formation was assessed using the In Vitro Angiogenesis assay kit as previously described (15). Briefly, HLECs were treated with PZH and/or exogenous VEGF-C for $24 \mathrm{~h}$, harvested and diluted $2 \times 10^{5}$ cells/well in $200 \mu \mathrm{l}$ medium individually, were seeded into 1:1 ECMatix gel (v/v) coated 48-well plate, and incubated for $8 \mathrm{~h}$. The series of tube-like structures were photographed using a phase-contrast microscope at a magnification of $\mathrm{x} 40$.

Western blot analysis. Cells were treated with PZH and/or exogenous VEGF-C for $24 \mathrm{~h}$ after which they were lysed using Pierce RIPA buffer containing protease inhibitor and phosphatase inhibitor cocktails. The lysates were then centrifuged at $14,000 \mathrm{rpm}$ for $20 \mathrm{~min}$, and the resulting protein concentrations were determined using BCA protein assay reagent kit. A total of $50 \mu \mathrm{g}$ of protein for each sample was loaded onto a $10 \%$ sodium dodecyl sulfate-polyacrylamide gel electrophoresis (SDS-PAGE) and resolved using $20 \mathrm{~V}$ for $10 \mathrm{~min} \rightarrow 80 \mathrm{~V}$ for $30 \mathrm{~min} \rightarrow 120 \mathrm{~V}$ for $1 \mathrm{~h}$, then transferred onto nitrocellulose membranes. Following blocking with 5\% non-fat dry milk, the membranes were incubated with antibodies against VEGF-C, VEGFR-3, MMP-2, MMP-9 and/or $\beta$-actin (1:1,000 dilution) overnight at $4{ }^{\circ} \mathrm{C}$ and subsequently incubated with HRP-conjugated anti-rabbit secondary antibodies (1:5,000 dilution) for $1 \mathrm{~h}$ at room temperature. The membranes were then exposed with enhanced chemiluminescence (ECL) 


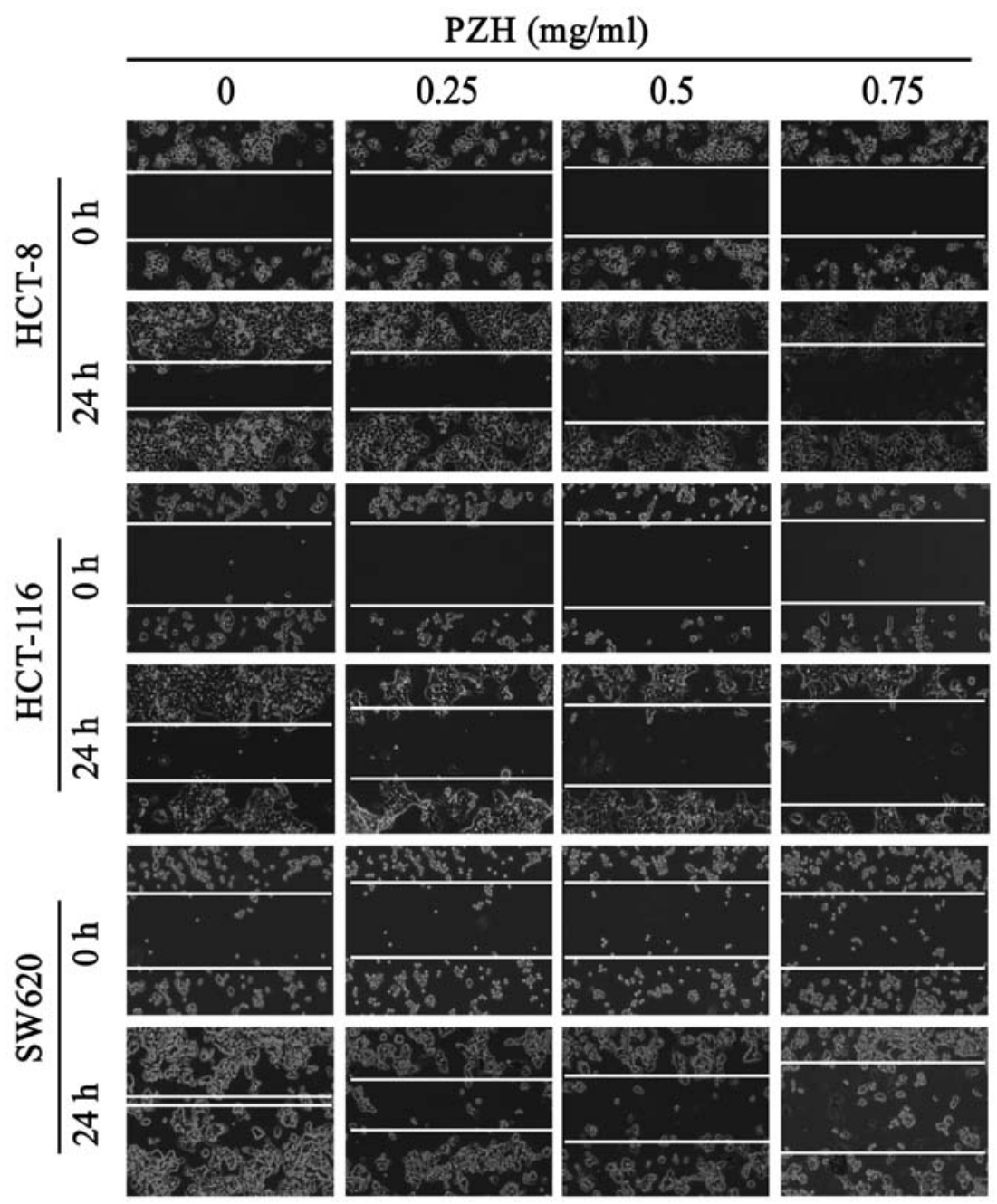

Figure 2. PZH reduces migration of CRC cell lines. HCT-8, HCT-116 and SW620 cells were treated with the indicated concentrations of PZH for 24 h. The images of wound healing are representative images of three independent experiments, and were captured at a magnification of x200.

detection using SuperSignal ${ }^{\mathrm{TM}}$ West Pico Chemiluminescent Substrate. Image Lab $^{\text {TM }}$ Software (version 3.0) was used for densitometric analysis and quantification of western blot analyses.

Statistical analysis. All data were collected based on the mean of three experiments. Statistical analysis was performed using the SPSS software (version 17.0) for Windows (SPSS, Inc., Chicago, IL, USA) using one-way ANOVA. P<0.05 was considered to indicate a statistically significant result.

\section{Results}

$P Z H$ reduces viability and migration of $C R C$ cell lines. We first determined the effect of $\mathrm{PZH}$ on the viability of various CRC cell lines using MTS assay. PZH treatment at 0.25 , 0.5 and $0.75 \mathrm{mg} / \mathrm{ml}$ for 24 or $48 \mathrm{~h}$ significantly reduced cell viability in the HCT-8, HCT-116 and SW620 cells $(\mathrm{P}<0.05)$ in a dose- and time-dependent manner (Fig. 1). We next evaluated the effect of $\mathrm{PZH}$ on migration of various CRC cell lines using a wound-healing assay. Twenty-four hours post-wounding, untreated HCT-8, HCT-116 and SW620 cells migrated into the wounded (clear) area of the cell monolayer, whereas $\mathrm{PZH}$ treatment dose-dependently inhibited migration of all these three cell lines (Fig. 2).

PZH downregulates the expression of VEGF-C in CRC cell lines. To explore a potential mechanism underlying the antimetastasis activity of PZH, we performed western blotting to examine the protein expression of VEGF-C in HCT-8, HCT-116 and SW620 cells. We found that PZH treatment profoundly and dose-dependently reduced the expression of VEGF-C (Fig. 3).

$P Z H$ reduces cell confluency and viability of VEGF-Cstimulated HLECs. As seen from the cell morphology, VEGF-C stimulation increased the confluency of HLECs, while $\mathrm{PZH}$ treatment decreased cell confluency in a dosedependent manner (Fig. 4A). Moreover, we found that cell viability of HLECs increased to $105.56 \%$ at $24 \mathrm{~h}$ and $113.56 \%$ by $48 \mathrm{~h}(\mathrm{P}<0.05)$ after VEGF-C stimulation compared to control cells that did not receive VEGF-C, using an MTS assay. Treatment with $0.0625-0.5 \mathrm{mg} / \mathrm{ml}$ of $\mathrm{PZH}$ for $24 \mathrm{~h}$ decreased the viability of VEGF-C-stimulated cells from 97.94 to $35.07 \%$ and from 81.01 to $24.10 \%$ after $48 \mathrm{~h}(\mathrm{P}<0.05$ vs. PZH-untreated cells) (Fig. 4B). 
A

\begin{tabular}{cccc}
\multicolumn{3}{c}{ PZH $(\mathrm{mg} / \mathrm{ml})$} \\
\hline 0 & 0.25 & 0.5 & 0.75
\end{tabular}
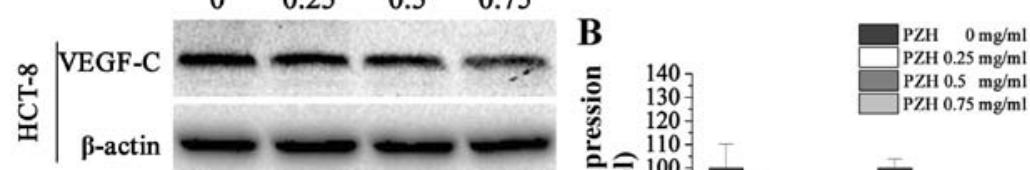

\begin{tabular}{l|l} 
VEGF-C & \\
岁-actin
\end{tabular}

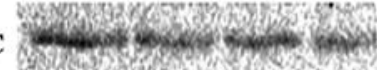

in

癹 $\mid \begin{aligned} & \text { VEGF-C } \\ & \beta \text {-actin }\end{aligned}$
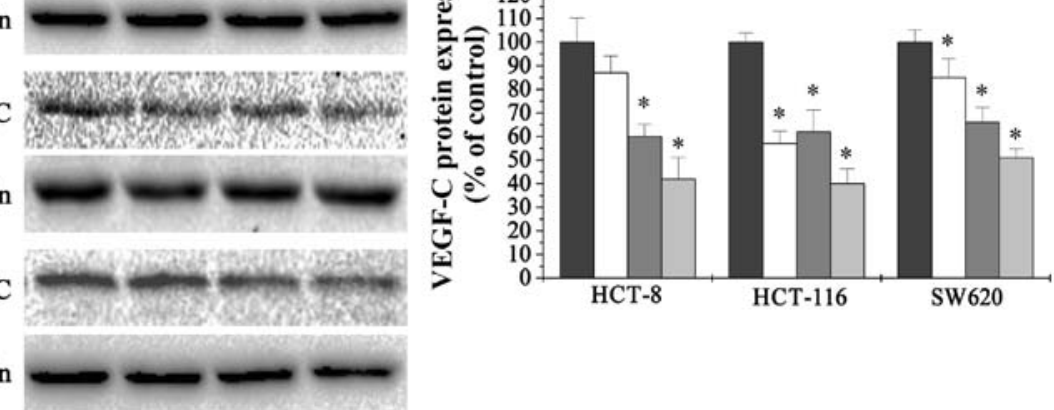

Figure 3. PZH downregulates the expression of VEGF-C in CRC cell lines. The protein expression levels of VEGF-C were analyzed by western blotting $\beta$-actin was used as the internal control. (A) Data are representative of three independent experiments. (B) Quantification of western blot analysis; ${ }^{2} \mathrm{P}<0.05$, vs. the untreated control cells.

A
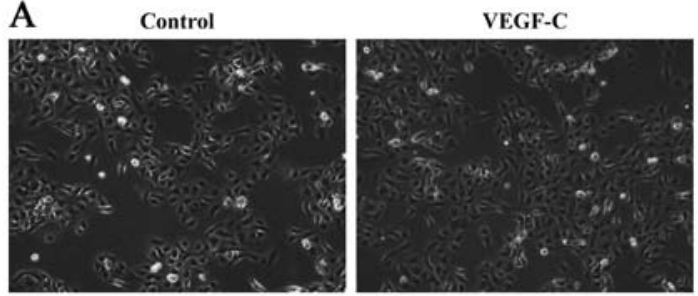

VEGF-C+PZH $0.0625 \mathrm{mg} / \mathrm{ml}$

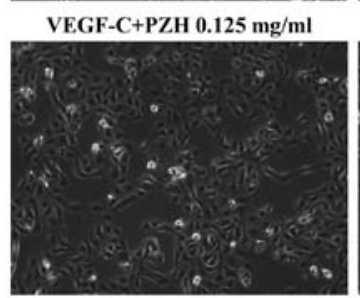

VEGF-C+PZH $0.25 \mathrm{mg} / \mathrm{ml}$
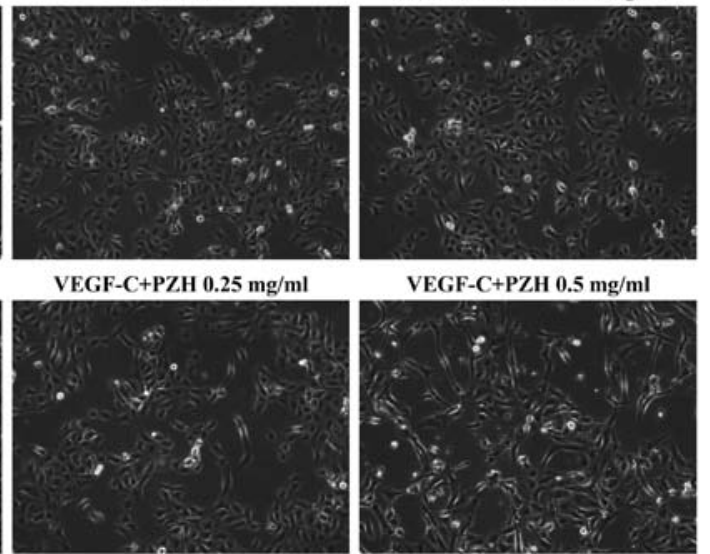

VEGF-C+PZH $0.5 \mathrm{mg} / \mathrm{ml}$
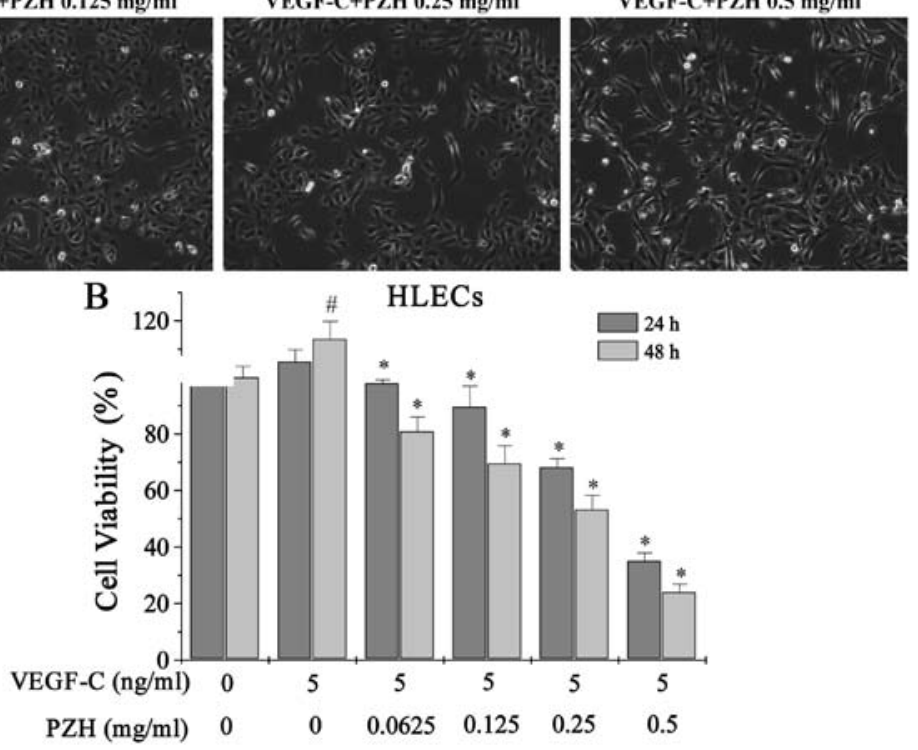

Figure 4. PZH reduces the cell confluency and viability of VEGF-C-stimulated HLECs. (A) HLECs were treated with exogenous VEGF-C or the indicated concentrations of PZH for $24 \mathrm{~h}$; cell confluency and morphology are representative images of three independent experiments, captured at a magnification of x200. (B) Cell viability was determined by MTS assays. The data were normalized to the viability of the control cells. Data are averages with SD (error bars) from at least three independent experiments; ${ }^{~} \mathrm{P}<0.05$, vs. the control cells; ${ }^{*} \mathrm{P}<0.05$, vs. the $\mathrm{PZH}$-untreated cells.

PZH does not affect the cell apoptosis of VEGF-C-stimulated HLECs. The effect of PZH on apoptosis in the VEGF-Cstimulated HLECs was determined by Annexin V/PI staining followed by FACS analysis. The results indicated that the percentage of cells undergoing apoptosis after treatment with $0.0625,0.125$ or $0.25 \mathrm{mg} / \mathrm{ml}$ of PZH (including the early and late apoptotic cells) was not significantly different when compared with the control cells that did not receive PZH treatment (Fig. 5).

PZH attenuates cell migration and tube formation ability of $V E G F$-C-stimulated HLECs. Transwell assays were performed to determine the effects of PZH on migration of HLECs. VEGF-C stimulated cells had enhanced migratory capacity 
A

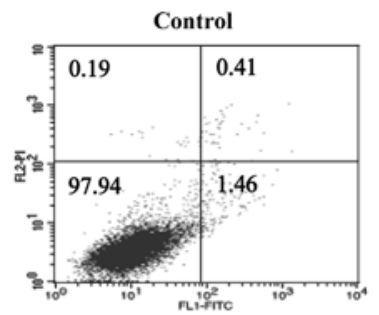

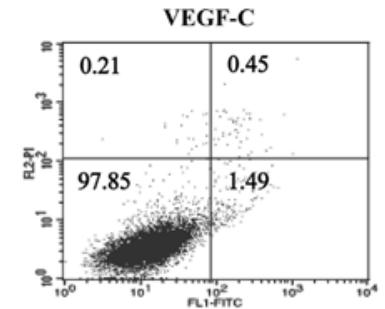
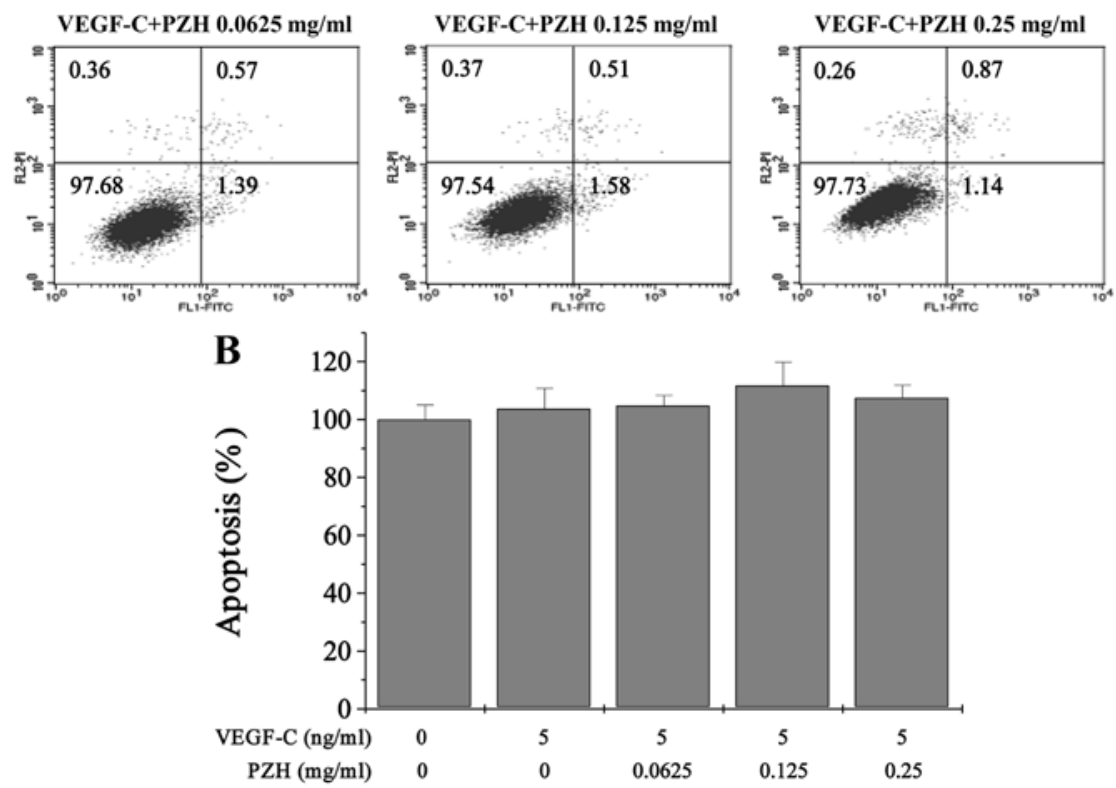

Figure 5. PZH not affects cell apoptosis of VEGF-C stimulated HLECs. (A) FACS analysis with Annexin V/PI staining. (B) Quantification of FACS analysis.

A

Control

VEGF-C
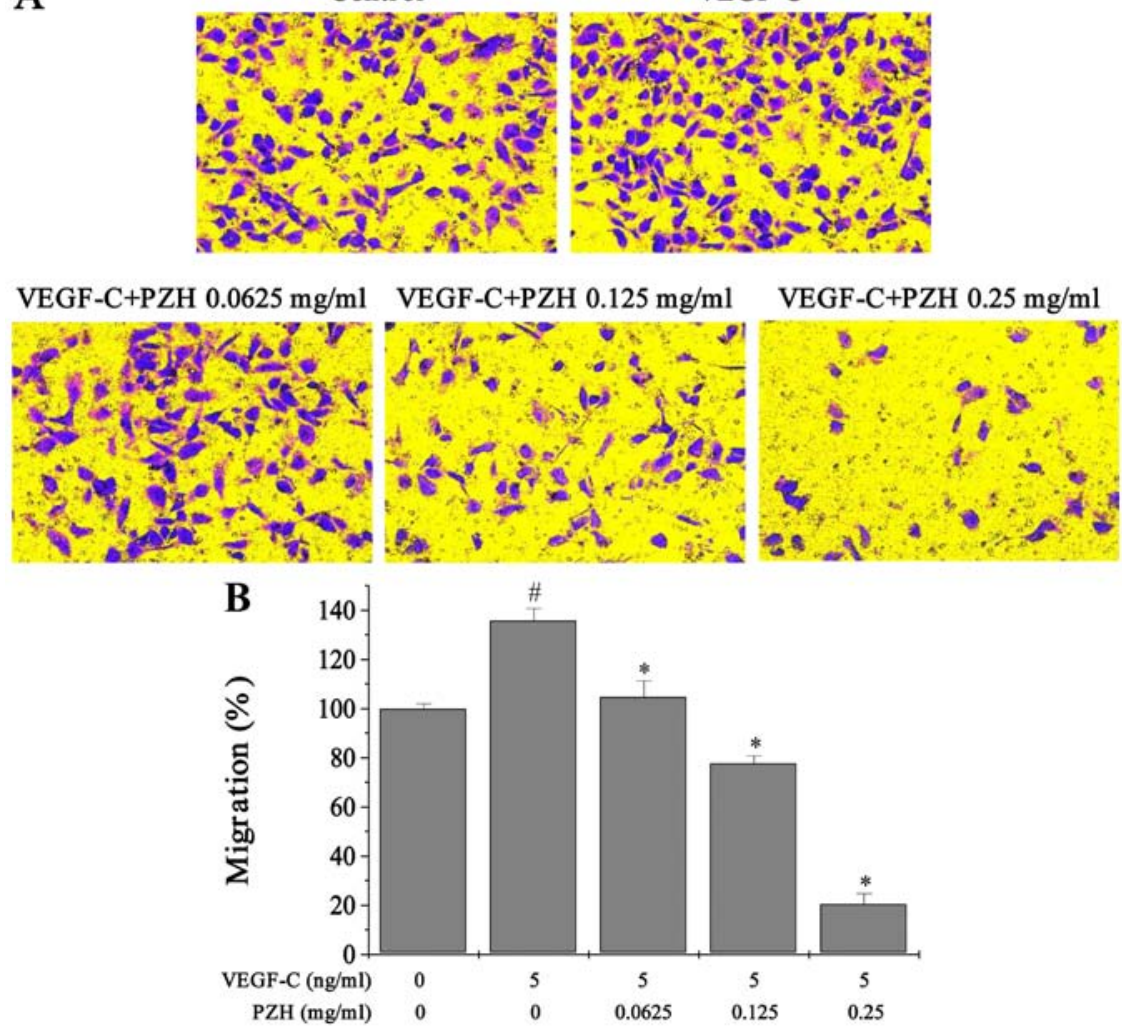

Figure 6. PZH attenuates cell migration ability of VEGF-C-stimulated HLECs. (A) The cell migration ability of HLECs was evaluated using Transwell cell culture chambers. Cells were stained with crystal violet; the images were captured at a magnification of x200. (B) The average number of migrated cells was counted in five randomly selective fields. The data were normalized to the migration of the control cells (100\%). Data are averages with SD (error bars) from three independent experiments; ${ }^{*} \mathrm{P}<0.05$, vs. the control cells; ${ }^{*} \mathrm{P}<0.05$, vs. the $\mathrm{PZH}$-untreated cells. 
A
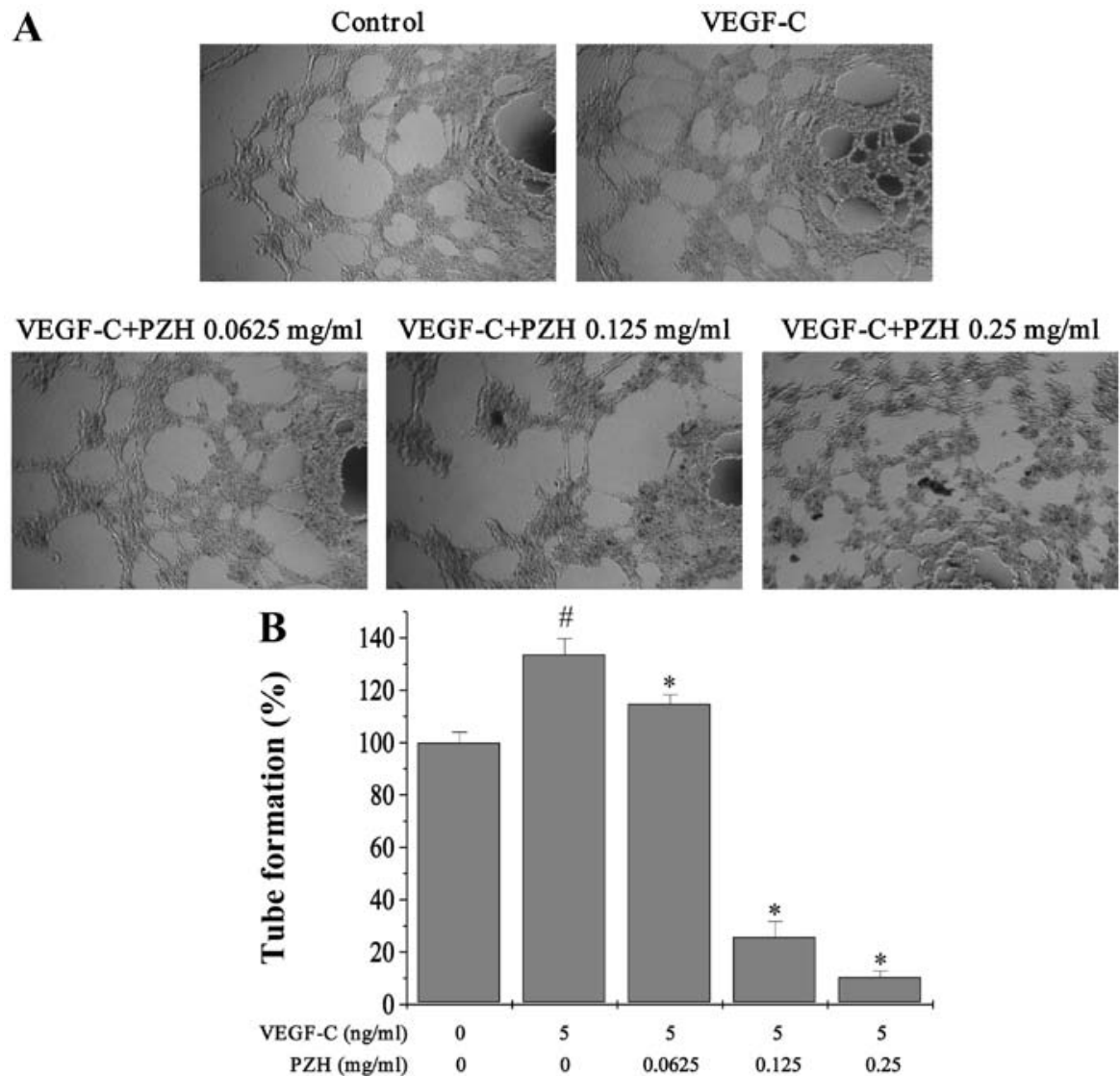

Figure 7. PZH attenuates tube formation ability of VEGF-C-stimulated HLECs. (A) The tube formation ability of HLECs was evaluated using the In Vitro Angiogenesis Assay kit. The images were captured at a magnification of $\mathrm{x} 40$. (B) The average number of formed tubes was counted in five randomly selective fields. The data were normalized to the formed tubes of control cells (100\%). Data are averages with SD (error bars) from three independent experiments;

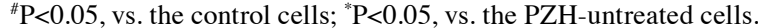

A

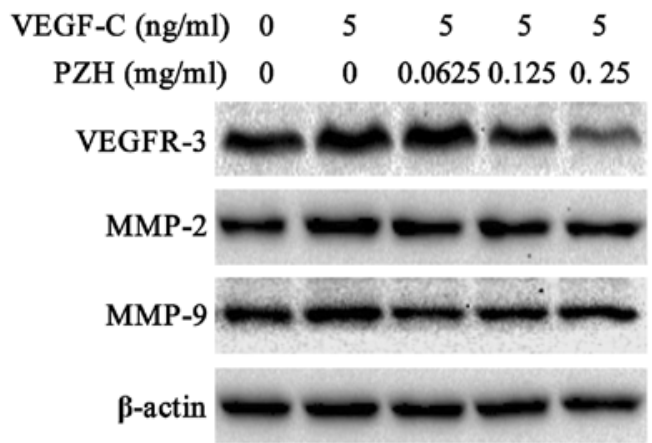

B

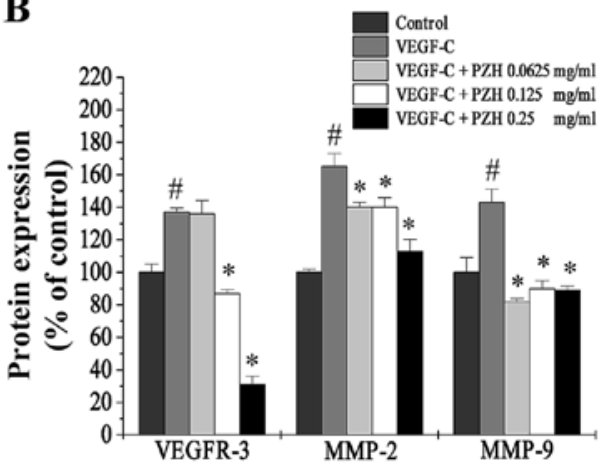

Figure 8. PZH downregulates the expression of VEGFR-3, MMP-2 and MMP-9 in VEGF-C-stimulated HLECs. The protein expression levels of VEGFR-3, MMP-2 and MMP-9 were analyzed by western blotting. $\beta$-actin was used as the internal control. (A) Data are representative of three independent experiments. (B) Quantification of western blot analysis; ${ }^{\text {P }}<0.05$, vs. the control cells; ${ }^{*} \mathrm{P}<0.05$, vs. the $\mathrm{PZH}$-untreated cells.

by $\sim 35.74 \%(\mathrm{P}<0.0327)$. Treatment with $0.0625-0.25 \mathrm{mg} / \mathrm{ml}$ of PZH dose-dependently reduced the cell migratory ability by $104.81-20.49 \%(\mathrm{P}<0.05)$ (Fig. 6). Similarly, exogenous VEGF-C stimulation led to a $33.69 \%(\mathrm{P}<0.0218)$ increase in tube formation of HLECs, compared to control cells that did not receive VEGF-C, which in turn was decreased by PZH treatment by $114.81-10.49 \%(\mathrm{P}<0.05)($ Fig. 7$)$.

PZH downregulates the expression of VEGFR-3, MMP-2 and MMP-9 in VEGF-C-stimulated HLECs. VEGFR-3 is the cognate receptor of VEGF-C that is expressed in HLECs. Binding of VEGF-C to VEGFR-3 results in proliferation of HLECs as well as the formation of LVs. Moreover, activation of the VEGF-C/VEGFR-3 signaling pathway can lead to the upregulation of matrix metalloproteinases (MMPs). Expression of MMP-2 and MMP-9 has been associated with the migration ability of VEGF-C-stimulated cells and lymphangiogenesis (20). To further explore the anti-lymphangiogenesis properties of $\mathrm{PZH}$, we used western blot analysis to determine the relative expression of these proteins after PZH 
treatment. We found that VEGFR-3, MMP-2 and MMP-9 were upregulated after VEGF-C stimulation and downregulated with PZH treatment (Fig. 8).

\section{Discussion}

The growth and metastatic spread of malignant tumors is critically dependent on the development of new vasculature. Lymphangiogenesis is strongly associated with tumor metastasis and has therefore been evaluated in various types of tumor including colon malignancies (5), esophageal carcinoma (21) and breast cancer (22). Recently, numerous studies have cast new light on the process of lymphangiogenesis and potential molecular mechanisms underlying tumor regional lymph node metastasis. One such mechanism is tumor-induced lymphangiogenesis. Evidence of intratumoral LVs has raised the possibility that tumor cells themselves contribute to lymphatic metastasis through the induction of lymphangiogenic processes (23). It is well known that tumor cells enter the lymphatic vasculature by eliciting lymphangiogenesis via growth factor production. Moreover, lymphangiogenic growth factors produced by tumor cells stimulate growth and dilation of the tumor-induced LVs as well as facilitate tumor regional lymph node metastasis.

VEGF-C, a lymphangiogenic growth factor, is a key regulator of lymphangiogenesis and tumor metastasis (24). Early studies have suggested that VEGF-C can promote the growth of new LVs and regional metastasis by binding to their receptor tyrosine kinase VEGFR-3, which was found to be densely expressed in lymphatic endothelial cells $(25,26)$. Studies with human or animal tumor models have demonstrated that malignant tumor cells themselves can secrete high levels of VEGF-C (27), and this overexpression of tumor-derived VEGF-C may play an important role in the occurrence of intratumoral lymphangiogenesis leading to the dissemination of tumor cells to regional lymph nodes (28-30).

Targeting lymphangiogenesis and VEGF-C may be a new treatment strategy for CRC. However, gene therapy targeting VEGF-C or the use of VEGFR-3 receptor antagonist has many disadvantages in clinical practice and can lead to drugresistance or other side-effects. For this reason, the advantage of traditional Chinese medicine (TCM) has been fully recognized. TCM formulas consist of a combination of many natural products, each of which contains numerous chemical compounds. Therefore, TCM prescription is often considered to have multi-component and multi-target effect. Due to the broad range of therapeutic functions of TCM, it has long been used to treat various diseases. One such prescription is Pien Tze Huang $(\mathrm{PZH})$, which exhibits specific anticancer activities.

In the present study, we demonstrated that PZH significantly reduced viability and migration in different CRC cell lines, which indicated that $\mathrm{PZH}$ possesses markedly antimetastasis ability (Figs. 1 and 2). To better understand its underlying mechanism, we determined the expression of VEGF-C in different $\mathrm{CRC}$ cell lines and found that $\mathrm{PZH}$ markedly downregulated VEGF-C expression. This suggests a potential mechanism, that the inhibition of VEGF-C expression is associated with the lymphangiogenesis-suppressive effects of PZH (Fig. 3). Since VEGF-C secreted from CRC cells can lead to the growth of HLECs and the subsequent formation of lymph vessels, we used exogenous VEGF-C stimulation of HLECs to establish a model of these processes in vitro. As shown in the results, $5 \mathrm{ng} / \mathrm{ml}$ of exogenous VEGF-C stimulation significantly enhanced cell confluency, viability and the ability of migration and tube formation, while $\mathrm{PZH}$ treatment attenuated all of these effects (Figs. 4 and 6). Notably, we found no change in cell apoptosis in response to PZH treatment (Fig. 5), which suggests that $\mathrm{PZH}$ suppresses lymphangiogenesis mainly by the inhibition of cell proliferation, migration and tube formation but not directly by promoting apoptosis of HLECs. Next, to further investigate the mechanisms of the anticancer effects of PZH on VEGF-C stimulated HLECs, we determined its effect on the expression of related proteins. VEGFR-3 is the cognate receptor to VEGF-C and is expressed in HLECs. PZH treatment notably downregulated the expression of VEGFR-3, which was increased by exogenous VEGF-C stimulation. Moreover, the VEGF-C/VEGFR-3 signaling pathway can lead to the activation of downstream transcription factors, resulting in the upregulation of MMPs, which is important for cell migration. Particularly, the increase in MMP-2 and MMP-9 can lead to the breakdown of extracellular matrix (ECM) in the surrounding tumor tissue and allow for the migration of HLECs. However, MMP-2 and MMP-9 can trigger the release of VEGF-C resulting in the proliferation of HLECs and the formation or remodeling of LVs $(31,32)$. We found that PZH prominently downregulated the expression of MMP-2 and MMP-9, which was increased by exogenous VEGF-C stimulation. Overall, these results demonstrate that $\mathrm{PZH}$ suppression of lymphangiogenesis via the downregulation of VEGF-C may be a molecular mechanism by which $\mathrm{PZH}$ inhibits metastasis in CRC.

In conclusion, $\mathrm{PZH}$ exerts its antimetastatic activities through the suppression of VEGF-C-mediated lymphangiogenesis, and thus may be developed as a promising multi-potent anticancer agent for the clinical treatment of CRC.

\section{Acknowledgements}

The present study was sponsored by the Natural Science Foundation of Fujian Province, China (no. 2015J01337).

\section{References}

1. Siegel RL, Miller KD and Jemal A: Cancer statistics, 2016. CA Cancer J Clin 66: 7-30, 2016.

2. Becouarn Y and Rougier P: Clinical efficacy of oxaliplatin monotherapy: Phase II trials in advanced colorectal cancer. Semin Oncol 25 (Suppl 5): S23-S31, 1998.

3. Ng M, Roy-Chowdhury S, Lum SS, Morgan JW and Wong JH: The impact of the ratio of positive to total lymph nodes examined and outcome in colorectal cancer. Am Surg 75: 873-876, 2009.

4. Jemal A, Clegg LX, Ward E, Ries LA, Wu X, Jamison PM, Wingo PA, Howe HL, Anderson RN and Edwards BK: Annual report to the nation on the status of cancer, 1975-2001, with a special feature regarding survival. Cancer 101: 3-27, 2004.

5. Sundlisaeter E, Dicko A, Sakariassen PØ, Sondenaa K, Enger PØ and Bjerkvig R: Lymphangiogenesis in colorectal cancer - prognostic and therapeutic aspects. Int J Cancer 121: 1401-1409, 2007.

6. Onogawa S, Kitadai Y, Tanaka S, Kuwai T, Kimura S and Chayama K: Expression of VEGF-C and VEGF-D at the invasive edge correlates with lymph node metastasis and prognosis of patients with colorectal carcinoma. Cancer Sci 95: 32-39, 2004. 
7. Kim JG, Chae YS, Sohn SK, Cho YY, Moon JH, Park JY, Jeon SW, Lee IT, Choi GS and Jun SH: Vascular endothelial growth factor gene polymorphisms associated with prognosis for patients with colorectal cancer. Clin Cancer Res 14: 62-66, 2008.

8. Parr C and Jiang WG: Quantitative analysis of lymphangiogenic markers in human colorectal cancer. Int J Oncol 23: 533-539, 2003.

9. Royston D and Jackson DG: Mechanisms of lymphatic metastasis in human colorectal adenocarcinoma. J Pathol 217: 608-619, 2009.

10. Folkman J: Role of angiogenesis in tumor growth and metastasis. Semin Oncol 29 (Suppl 16): S15-S18, 2002.

11. Hsu JY and Wakelee HA: Monoclonal antibodies targeting vascular endothelial growth factor: Current status and future challenges in cancer therapy. BioDrugs 23: 289-304, 2009.

12. Chinese Pharmacopoeia Commission. Pharmacopoeia of the Peoples Republic of China. Vol. 1. Chinese Medical Science and Technology Press, Beijing, pp573-575, 2010.

13. Shen A, Hong F, Liu L, Lin J, Wei L, Cai Q, Hong Z and Peng J: Pien Tze Huang inhibits the proliferation of human colon carcinoma cells by arresting G1/S cell cycle progression. Oncol Lett 4: 767-770, 2012.

14. Lin JM, Wei LH, Chen YQ, Liu XX, Hong ZF, Sferra TJ and Peng J: Pien Tze Huang induced apoptosis in human colon cancer HT-29 cells is associated with regulation of the Bcl-2 family and activation of caspase 3. Chin J Integr Med 17: 685-690, 2011.

15. Shen AL, Hong F, Liu LY, Lin JM, Zhuang QC, Hong ZF and Peng J: Effects of Pien Tze Huang on angiogenesis in vivo and in vitro. Chin J Integr Med 18: 431-436, 2012.

16. Wei L, Chen P, Chen Y, Shen A, Chen H, Lin W, Hong Z, Sferra TJ and Peng J: Pien Tze Huang suppresses the stem-like side population in colorectal cancer cells. Mol Med Rep 9: 261-266, 2014.

17. Shen A, Lin J, Chen Y, Lin W, Liu L, Hong Z, Sferra TJ and Peng J: Pien Tze Huang inhibits tumor angiogenesis in a mouse model of colorectal cancer via suppression of multiple cellular pathways. Oncol Rep 30: 1701-1706, 2013.

18. Shen A, Chen H, Chen Y, Lin J, Lin W, Liu L, Sferra TJ and Peng J: Pien Tze Huang overcomes multidrug resistance and epithelial-mesenchymal transition in human colorectal carcinoma cells via suppression of TGF- $\beta$ pathway. Evid Based Complement Alternat Med 2014: 679436, 2014.

19. Lin W, Zhuang Q, Zheng L, Cao Z, Shen A, Li Q, Fu C, Feng J and Peng J: Pien Tze Huang inhibits liver metastasis by targeting TGF- $\beta$ signaling in an orthotopic model of colorectal cancer. Oncol Rep 33: 1922-1928, 2015.
20. Grau S: Expression of lymphangiogenesis-related factors in malignant gliomas. Neurosci Res 60: 40-49, 2008.

21. Saad RS, Lindner JL, Liu Y and Silverman JF: Lymphatic vessel density as prognostic marker in esophageal adenocarcinoma. Am J Clin Pathol 131: 92-98, 2009.

22. Liu HT, Ma R, Yang QF, Du G and Zhang CJ: Lymphangiogenic characteristics of triple negativity in node-negative breast cancer. Int J Surg Pathol 17: 426-431, 2009.

23. Oliver G and Detmar M: The rediscovery of the lymphatic system: Old and new insights into the development and biological function of the lymphatic vasculature. Genes Dev 16: 773-783, 2002.

24. Caunt M, Mak J, Liang WC, Stawicki S, Pan Q, Tong RK, Kowalski J, Ho C, Reslan HB, Ross J, et al: Blocking neuropilin-2 function inhibits tumor cell metastasis. Cancer Cell 13: 331-342, 2008.

25. Bahram $\mathrm{F}$ and Claesson-Welsh L: VEGF-mediated signal transduction in lymphatic endothelial cells. Pathophysiology 17: 253-261, 2010.

26. Girling JE and Rogers PA: Regulation of endometrial vascular remodelling: Role of the vascular endothelial growth factor family and the angiopoietin-TIE signalling system. Reproduction 138: 883-893, 2009.

27. Saintigny P, Kambouchner M, Ly M, Gomes N, SainteCatherine O, Vassy R, Czernichow S, Letoumelin P, Breau JL, Bernaudin JF, et al: Vascular endothelial growth factor-C and its receptor VEGFR-3 in non-small-cell lung cancer: Concurrent expression in cancer cells from primary tumour and metastatic lymph node. Lung Cancer 58: 205-213, 2007.

28. Chang L, Kaipainen A and Folkman J: Lymphangiogenesis new mechanisms. Ann NY Acad Sci 979: 111-119, 2002.

29. Su JL, Chen PS, Chien MH, Chen PB, Chen YH, Lai CC, Hung MC and Kuo ML: Further evidence for expression and function of the VEGF-C/VEGFR-3 axis in cancer cells. Cancer Cell 13: 557-560, 2008.

30. Tammela T and Alitalo K: Lymphangiogenesis: Molecular mechanisms and future promise. Cell 140: 460-476, 2010.

31. Bergers G, Brekken R, McMahon G, Vu TH, Itoh T, Tamaki K, Tanzawa K, Thorpe P, Itohara S, Werb Z, et al: Matrix metalloproteinase-9 triggers the angiogenic switch during carcinogenesis. Nat Cell Biol 2: 737-744, 2000.

32. Rojiani MV, Alidina J, Esposito N and Rojiani AM: Expression of MMP-2 correlates with increased angiogenesis in CNS metastasis of lung carcinoma. Int J Clin Exp Pathol 3: 775-781, 2010. 